\title{
SPHERE-PACKING MODULATED SPACE-TIME CODING USING NON-BINARY LDPC-CODED ITERATIVE-DETECTION
}

\author{
O. Alamri ${ }^{1}$, S. X. $\mathrm{Ng}^{1}, \mathrm{~F} . \mathrm{Guo}^{1}, \mathrm{~S} . \mathrm{Zummo}^{2}$ and L. Hanzo ${ }^{1 *}$ \\ ${ }^{1}$ School of ECS, University of Southampton, SO17 1BJ, UK. \\ Email: 1h@ecs.soton.ac.uk \\ http: //www-mobile.ecs.soton.ac.uk \\ ${ }^{2}$ Dept. of Elec. Eng., King Fahd University of Pertoleum and Minerals, Dhahran, Saudi Arabia.
}

\begin{abstract}
A recently proposed Space-Time Block Coding (STBC) signal construction method that combines orthogonal design with sphere packing, referred to here as (STBC-SP), has shown useful performance improvements over Alamouti's conventional orthogonal design. In this contribution, we propose a purely symbol-based LDPC-coded scheme, demonstrating that the performance of STBC-SP systems can be further improved by concatenating sphere packing aided modulation with non-binary LDPC and by performing symbol-based turbo detection between the non-binary LDPC decoder and a rate-1 non-binary inner precoder. We also investigate the convergence behaviour of this symbolbased concatenated scheme with the aid of novel nonbinary Extrinsic Information Transfer (EXIT) charts. Finally, we demonstrate that in the investigated scenarios it requires 1-2dB lower power in comparison to equivalent effectivethroughput $0.5,0.75$ and 1 bit/symbol systems employing bit-based turbo detection.
\end{abstract}

\section{INTRODUCTION}

The adverse effects of channel fading may be significantly reduced by employing space-time coding invoking multiple antennas [1]. Alamouti [2] discovered an appealingly simple transmit diversity scheme employing two transmit antennas. This low-complexity design inspired Tarokh et al. [3] to generalise Alamouti's transmit diversity scheme using the principle of orthogonal design to an arbitrary number of transmit antennas. Since then, the pursuit of designing better space-time modulation schemes has attracted considerable further attention [4]. The concept of combining orthogonal transmit diversity designs with the principle of sphere packing was introduced by Su et al. in [5], where it was demonstrated that the proposed Sphere Packing (SP) aided Space-Time Block Coded (STBC) system, referred to here as STBC-SP, was capable of outperforming the conventional orthogonal design based STBC schemes of $[2,3]$.

Surprisingly, the family of Low Density Parity Check (LDPC) codes originally devised by Gallager as early as 1963 [6] remained more or less unexploited until after the discovery of turbo codes in 1993 [7]. Since then, however, LDPC codes have experienced a renaissance [8] and attracted substantial research interests. In 1998, Davey and MacKay proposed a non-binary version of LDPC codes [9], which was potentially capable of outperforming binary LDPC codes.

Similarly, the family of concatenated codes pioneered by Forney in 1966 [10] failed to generate as much research interest as it deserves, largely owing to its complexity. Nonetheless, the Consultative Committee for Space Data Systems (CCSDS) standardised an attractive combination of powerful inner convolutional coding and outer Reed-Solomon codes in [11], which refrained from iterative detection. However, upon the discovery of turbo codes [7], it was shown that efficient iterative decoding

\footnotetext{
* The financial support of the EPSRC, UK and that of the European Union as well as that of the Ministry of Higher Education of Saudi Arabia is gratefully acknowledged.
}

of concatenated codes can be carried out at a low complexity by employing simple constituent codes. Since then, the appealing iterative decoding of concatenated codes has inspired numerous authors to extend the technique to other transmission schemes consisting of a concatenation of two or more constituent detection/decoding stages [12-17]. For example, in [12], the turbo principle was extended to serially concatenated block and convolutional codes. It was also shown in [13] that a recursive inner code is needed in order to maximise the interleaver gain and hence to avoid a bit-error rate (BER) floor, when employing iterative decoding. This principle has been further adopted by several authors in designing serially concatenated schemes, where rate-1 inner codes were employed for creating low complexity turbo codes suitable for bandwidth and power limited systems having stringent BER requirements [14-16]. In [18], it was shown that a reduced transmit power may be required, when symbolbased rather than bit-based iterative decoding is employed.

Recently, studying the convergence behaviour of iterative decoding semi-analytically has attracted considerable attention [19$22]$, since their mathematical analysis remains impervious. In [19], ten Brink proposed the employment of the so-called extrinsic information transfer (EXIT) characteristics between a concatenated decoder's output and input for describing the flow of extrinsic information through the soft in/soft out constituent decoders. EXIT charts have been extended to the non-binary (indexbased) case in [20], where a histogram-based approximation of the extrinsic information was required in order to compute the mutual information. An efficient and low-complexity method of computing non-binary EXIT charts was proposed in [21], which may be considered a generalisation of the approach presented in [22].

Motivated by the performance improvements reported in [5], [9], [14] and [18], we propose a novel symbol-based iterative scheme. We will demonstrate that the proposed non-binary turbodetection aided STBC-SP scheme is capable of providing further performance improvements over both the STBC-SP scheme of [5] as well as over a bit-based LDPC-coded turbo-detected STBCSP scheme. The novel non-binary EXIT charts of [20,21] are employed for designing our non-binary scheme, which makes this paper distinct from [23] in addition to the comparison with an equivalent bit-based scheme. The rationale of the proposed architecture is explicit: (1) SP modulation maximises the coding advantage of the transmission scheme by jointly designing and detecting the SP symbols hosting the two time-slots' STBC symbols ${ }^{1}$; (2) the inner rate-1 encoder and its low-complexity recursive decoder beneficially distributes the extrinsic information without reducing the effective throughput, maximises the interleaver gain at a given length and hence avoids having a BER floor; and (3) symbol-based iterative decoding outperforms its bit-based counterpart.

This paper is organised as follows. A system overview is pre-

\footnotetext{
${ }^{1}$ By contrast, Alamouti detected two seemingly independent QPSK space-time symbols, although their amalgam constitutes a combined symbol.
} 
sented in Section II. Symbol-based iterative decoding is described in Section III. Section IV provides our EXIT chart analysis, while our simulation results are discussed in Section V. Finally, we conclude in Section VI.

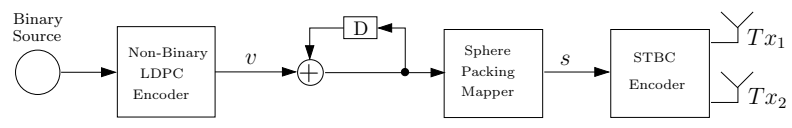

Fig. 1. Encoder of the symbol-based turbo detected STBC-SP scheme.

\section{SYSTEM OVERVIEW}

In this contribution, space-time systems employing two transmit antennas are considered, where the space-time signal is given by [2]

$$
\mathbf{G}_{2}\left(x_{1}, x_{2}\right)=\left[\begin{array}{cc}
x_{1} & x_{2} \\
-x_{2}^{*} & x_{1}^{*}
\end{array}\right]
$$

and the rows as well as columns of Equation (1) represent the temporal and spatial dimensions, corresponding to two consecutive time slots and two transmit antennas, respectively. In this section, we will describe the proposed turbo-detected symbolbased scheme as well as its bit-based counterpart. The schematic of the encoder of the non-binary arrangement is shown in Figure 1 . The source bits are encoded by a rate $R=\frac{1}{2}$ nonbinary LDPC encoder [9], to generate the LDPC encoded symbols $\mathbf{v}=\left\{v_{0}, v_{1}, \ldots, v_{K_{l d p c}-1}\right\}, v_{k} \in G F(q)$, where $K_{l d p c}$ is the LDPC output block length and $q$ is the size of the LDPC decoding field. The LDPC encoded symbols are then precoded by a non-binary rate- 1 encoder, before each of them is mapped to the corresponding sphere packing modulated symbol $\mathrm{s}^{l} \in S$, $0 \leq l \leq L-1$. More specifically, $S=\left\{\mathbf{s}^{l}=\left[\begin{array}{lll}a_{l, 1} & a_{l, 2} & a_{l, 3}\end{array}\right.\right.$ $\left.\left.a_{l, 4}\right] \in \mathbb{R}^{4}: 0 \leq l \leq L-1\right\}$ constitutes a set of $L$ legitimate constellation points selected from the lattice $D_{4}$ having a total energy of $E \triangleq \sum_{l=0}^{L-1}\left(\left|a_{l, 1}\right|^{2}+\left|a_{l, 2}\right|^{2}+\left|a_{l, 3}\right|^{2}+\left|a_{l, 4}\right|^{2}\right)$. There is a natural one-to-one mapping between $l$ and the elements of the non-binary LDPC code defined over $G F(q)$, where we have $L=q$, allowing us to create a purely symbol-based system. Again, the rate-1 precoder shown in Figure 1 is also a non-binary encoder, defined by the binary generator polynomial $G=\left(g / g_{r}\right)=(10 / 11)$, where $g$ denotes the feedforward output and $g_{r}$ is the feedback to the input using a modulo $q$ addition. Observe that channel interleaving is not required between the non-binary LDPC encoder and the rate- 1 encoder, since the LDPC parity check matrix is randomly constructed, where each of the parity check equations is checking several random $G F(q)$ symbol positions in a codeword, which has a similar effect to that of the channel interleaver. The STBC encoder then maps each sphere packing modulated symbol $\mathbf{s}^{l}$ to a space-time signal $C_{l}=\sqrt{\frac{2 L}{E}} \mathbf{G}_{2}\left(x_{l, 1}, x_{l, 2}\right), 0 \leq l \leq L-1$, such that $x_{l, 1}$ and $x_{l, 2}$ are written as

$$
\begin{aligned}
\left\{x_{l, 1}, x_{l, 2}\right\} & =T_{s p}\left(a_{l, 1}, a_{l, 2}, a_{l, 3}, a_{l, 4}\right) \\
& =\left\{a_{l, 1}+j a_{l, 2}, a_{l, 3}+j a_{l, 4}\right\} .
\end{aligned}
$$

Subsequently, each sphere-packed space-time coded symbol is transmitted over $T=2$ consecutive time slots using two transmit antennas, as shown in Equation (1). More detailed discussions on orthogonal design with sphere packing modulation are provided in [24].

In this treatise, we considered a correlated narrowband Rayleigh fading channel,associated with a normalised Doppler frequency of $f_{D}=f_{d} T_{s}=0.1$, where $f_{d}$ is the Doppler frequency and $T_{s}$ is the symbol period. The complex fading envelope is assumed to be constant across the transmission period of a spacetime coded symbol spanning $T=2$ time slots. The complex Additive White Gaussian Noise (AWGN) of $n=n_{I}+j n_{Q}$ is also added to the received signal, where $n_{I}$ and $n_{Q}$ are two independent zero mean Gaussian random variables having a variance of $\sigma_{n}^{2}=\sigma_{n_{I}}^{2}=\sigma_{n_{Q}}^{2}=N_{0} / 2$ per dimension, where $N_{0} / 2$ represents the double-sided noise power spectral density expressed in $\mathrm{W} / \mathrm{Hz}$.

As shown in Figure 2, the received complex-valued symbols are first decoded by the STBC decoder to produce a received sphere-packed symbol $\mathbf{r}$, which is fed into the sphere packing demapper, where the soft-metric $\mathbf{Q}(k)$ is calculated. More explicitly, the notation $\mathbf{Q}(k)$ represents the soft metric passed from the SP demapper to the non-binary rate-1 decoder based on the probability of the $k^{t h}$ symbol of the encoded codeword by the rate- 1 encoder, as will be shown in Section III. As seen in Figure 2, the rate- 1 decoder processes these soft-metrics in conjunction with the a priori information, $\mathbf{A}_{\text {urc }}$, in order to generate the $a$ posteriori probability, $\mathbf{D}_{\text {urc }}$, where the subscript urc refers to the unity-rate code. More specifically, the a priori information, $\mathbf{A}_{\text {urc }}$, is provided by the LDPC decoder as the soft metric for the LDPC encoded symbols. After removing the a priori information, $\mathbf{A}_{u r c}$, from the a posteriori probability denoted by $\mathbf{D}_{\text {urc }}$ using symbol-based element-wise division, as will be shown in Section III, $\mathbf{A}_{l d p c}$ is passed as a priori information to the LDPC decoder, which carries out a specified number of LDPC iterations and produces the decoded a posteriori probability $\mathbf{D}_{l d p c}$. Based on the a posteriori probability, a tentative hard decision will be made and the resultant codeword will be checked by the LDPC code's parity check matrix. If the resultant vector is an all-zero sequence, then a legitimate codeword has been found, and the hard-decision based sequence will be output. Otherwise, if the maximum affordable number of iterations has not been reached, the a priori information, $\mathbf{A}_{l d p c}$, is removed from the $a$ posteriori probability denoted by $\mathbf{D}_{l d p c}$ using symbol-based element-wise division and fed back to the non-binary rate-1 decoder for the next iteration, as seen in Figure 2. This process continues, until the affordable maximum number of iterations has been encountered or a legitimate codeword has been found.

The structure of the bit-based scheme is identical to its symbol-based counterpart seen in Figures 1 and 2, except that a binary - rather than non-binary - LDPC code is invoked in order to investigate the employment of bit interleaving and bitbased iterative decoding. Bit-to-symbol probability conversion is required, when passing extrinsic information from the binary LDPC decoder to the rate-1 decoder. On the other hand, symbolto-bit probability conversion is required, when passing extrinsic information from the rate- 1 decoder to the binary LDPC decoder. The bit probabilities at the input as well as at the output of the binary LDPC decoder are represented by their Log-Likelihood Ratios (LLRs).

\section{SYMBOL-BASED ITERATIVE DECODING}

For the sake of simplicity, a system having a single receive antenna is considered, although its extension to systems having more than one receive antenna is straightforward. Assuming perfect channel estimation, the complex-valued channel output symbols received during two consecutive time slots are first diversity-combined in order to extract the estimates $\tilde{x}_{1}$ and $\tilde{x}_{2}$ of the most likely transmitted symbols $x_{l, 1}$ and $x_{l, 2}$ [2][1, pp. $400-401]$, resulting in

$$
\begin{aligned}
& \tilde{x}_{1}=\left(\left|h_{1}\right|^{2}+\left|h_{2}\right|^{2}\right) \cdot x_{l, 1}+\dot{n}_{1} \\
& \tilde{x}_{2}=\left(\left|h_{1}\right|^{2}+\left|h_{2}\right|^{2}\right) \cdot x_{l, 2}+\dot{n}_{2},
\end{aligned}
$$

where $h_{1}$ and $h_{2}$ represent the complex-valued channel coefficients corresponding to the first and second transmit antenna, respectively, and $n_{1}$ as well as $n_{2}$ are zero-mean complex Gaussian random variables with variance $\sigma_{\dot{n}}^{2}=\left(\left|h_{1}\right|^{2}+\left|h_{2}\right|^{2}\right) \cdot \sigma_{n}^{2}$. A received sphere-packed symbol $\mathbf{r}$ is then constructed from the estimates $\tilde{x}_{1}$ and $\tilde{x}_{2}$ using Equation (2) as $\mathbf{r}=T_{s p}^{-1}\left(\tilde{x}_{1}, \tilde{x}_{2}\right)$, where $\mathbf{r}=\left\{\left[\begin{array}{llll}\tilde{a}_{1} & \tilde{a_{2}} & \tilde{a_{3}} & \tilde{a_{4}}\end{array}\right] \in \mathbb{R}^{4}\right\}$. The received sphere-packed symbol $\mathbf{r}$ can be written as

$$
\mathbf{r}=h \cdot \sqrt{\frac{2 L}{E}} \cdot \mathbf{s}^{l}+\mathbf{w}
$$




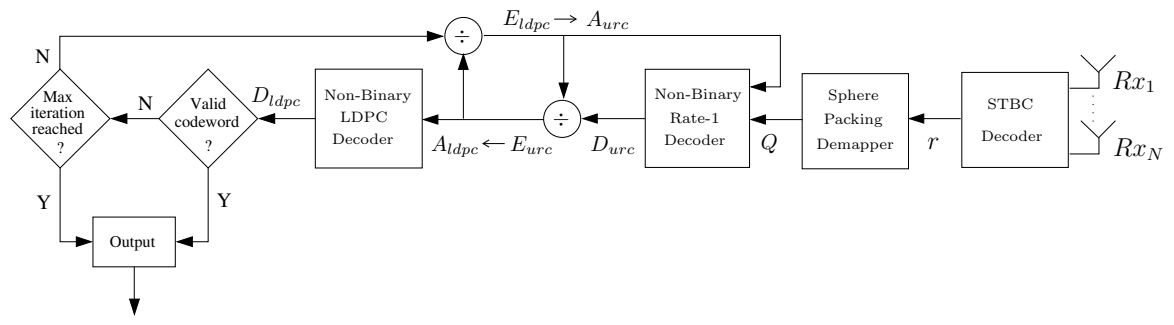

Fig. 2. Decoder of the symbol-based turbo detected STBC-SP scheme.

where $h=\left(\left|h_{1}\right|^{2}+\left|h_{2}\right|^{2}\right), \mathbf{s}^{l} \in S, 0 \leq l \leq L-1$, and $\mathbf{w}$ is a four-dimensional real-valued Gaussian random variable having a covariance matrix of $\sigma_{w}^{2} \cdot \mathbf{I}_{N_{D}}=\sigma_{\tilde{n}}^{2} \cdot \mathbf{I}_{N_{D}}=h \cdot \sigma_{n}^{2} \cdot \mathbf{I}_{N_{D}}$, where $N_{D}=4$, since the symbol constellation $S$ is four-dimensional. According to Equation (5), the conditional PDF $p\left(\mathbf{r} \mid \mathbf{s}^{l}\right)$ is given by

$$
\begin{aligned}
p\left(\mathbf{r} \mid \mathbf{s}^{l}\right) & =\frac{1}{\left(2 \pi \sigma_{w}^{2}\right)^{\frac{N_{D}}{2}}} e^{-\frac{1}{2 \sigma_{w}^{2}}\left(\mathbf{r}-\alpha \cdot \mathbf{s}^{l}\right)\left(\mathbf{r}-\alpha \cdot \mathbf{s}^{l}\right)^{T}}, \\
& =\frac{1}{\left(2 \pi \sigma_{w}^{2}\right)^{\frac{N_{D}}{2}}} e^{-\frac{1}{2 \sigma_{w}^{2}}\left(\sum_{i=1}^{4}\left(\tilde{a}_{i}-\alpha \cdot a_{l, i}\right)^{2}\right)},
\end{aligned}
$$

where we have $\alpha=h \cdot \sqrt{\frac{2 L}{E}}$ and $(\cdot)^{T}$ represents the transpose of a vector.

Similarly, the conditional PDF $p\left(\mathbf{s}^{l} \mid \mathbf{r}\right)$ is given by

$$
p\left(\mathbf{s}^{l} \mid \mathbf{r}\right)=\frac{p\left(\mathbf{r} \mid \mathbf{s}^{l}\right) \cdot p\left(\mathbf{s}^{l}\right)}{p(\mathbf{r})} .
$$

Since the LDPC codeword consists of $K_{l d p c} G F(q)$ symbols, the sphere packing demapper of Figure 2 will process $K_{l d p c}$ received sphere-packed symbols, $\left(\mathbf{r}_{0}, \mathbf{r}_{1}, \ldots, \mathbf{r}_{K_{l d p c}-1}\right)$, at a time to produce the following $\left(K_{l d p c} \times L\right)$ soft-metric matrix using Equation (7)

$$
\mathbf{Q}=\left[\begin{array}{llll}
\mathbf{Q}(0) & \mathbf{Q}(1) & \cdots & \mathbf{Q}\left(K_{l d p c}-1\right)
\end{array}\right]^{T},
$$

where,

$$
\mathbf{Q}(k)=\left[\begin{array}{llll}
p\left(\mathbf{s}_{k}=\mathbf{s}^{0} \mid \mathbf{r}_{k}\right) & p\left(\mathbf{s}_{k}=\mathbf{s}^{1} \mid \mathbf{r}_{k}\right) & \cdots & p\left(\mathbf{s}_{k}=\mathbf{s}^{L-1} \mid \mathbf{r}_{k}\right)
\end{array}\right],
$$

for $k=0,1, \ldots, K_{l d p c}-1$. All the probabilities corresponding to a specific row in $\mathbf{Q}$, which correspond to a specific received symbol, should be normalised so that they sum up to unity.

The non-binary rate- 1 decoder of Figure 2 then processes the soft-metric matrix $\mathbf{Q}$ of Equation (8) in conjunction with the a priori information, $\mathbf{A}_{u r c}$, in order to produce a decoded a posteriori probability matrix, $\mathbf{D}_{u r c}$, of size $\left(K_{l d p c} \times L\right)$ using a standard implementation of the forward-backward recursion based a posteriori probability (APP) algorithm ${ }^{2}$ [26]. During the first iteration, $p\left(\mathbf{s}^{l}\right), 0 \leq l \leq L-1$, seen in Equation (7) has to be set to $1 / q$, since no a priori information is yet available from the LDPC decoder. The a priori knowledge fed into the rate- 1 decoder of Figure 2 is removed from the decoded a posteriori probability matrix, $\mathbf{D}_{\text {urc }}$, using symbolbased element-wise division [20] for the sake of generating the extrinsic probability matrix, $\mathbf{E}_{u r c}$, which is then fed into the LDPC decoder as the a priori knowledge, $\mathbf{A}_{l d p c}$, as alluded to before. More specifically, the following $\left(K_{l d p c} \times L\right)$ a priori information matrix is constructed

$\mathbf{A}_{l d p c}=\left[\begin{array}{llll}\mathbf{A}_{l d p c}(0) & \mathbf{A}_{l d p c}(1) & \cdots & \mathbf{A}_{l d p c}\left(K_{l d p c}-1\right)\end{array}\right]^{T}$,

where,

\footnotetext{
${ }^{2}$ The APP terminology is used here rather than the Maximum $A$ posteriori Propability (MAP), since the soft-input soft-output rate1 decoder is computing probabilities rather than their maximum [25].
}

$$
\mathbf{A}_{l d p c}(k)=\left[\begin{array}{llll}
p\left(\mathbf{s}_{k}=\mathbf{s}^{0}\right) & p\left(\mathbf{s}_{k}=\mathbf{s}^{1}\right) & \cdots & p\left(\mathbf{s}_{k}=\mathbf{s}^{L-1}\right)
\end{array}\right],
$$

and we have

$p\left(\mathbf{s}_{k}=\mathbf{s}^{l}\right)=\frac{\left(d_{u r c}\right)_{k}^{l}}{\left(a_{u r c}\right)_{k}^{l}}, \quad 0 \leq l \leq L-1, \quad 0 \leq k \leq K_{l d p c}-1$,

while $\left(d_{u r c}\right)_{k}^{l}$ as well as $\left(a_{u r c}\right)_{k}^{l}$ refer to the elements at the cross-over point of the $k^{t h}$ row and $l^{t h}$ column of the matrices $\mathbf{D}_{u r c}$ and $\mathbf{A}_{u r c}$, respectively. Again, the probabilities corresponding to a specific row of the matrix $\mathbf{A}_{l d p c}$ should be normalised, so that the values add up to unity. The LDPC decoder exploits the a priori information, $\mathbf{A}_{l d p c}$, for the sake of producing a decoded soft-metric, $\mathbf{D}_{l d p c}$. Again, the a priori information, $\mathbf{A}_{l d p c}$, is removed from the decoded a posteriori probability matrix, $\mathbf{D}_{l d p c}$, by symbol-based element-wise division for the sake of generating $\mathbf{E}_{l d p c}$, which is passed to the rate-1 decoder of Figure 2 as the a priori knowledge, $\mathbf{A}_{u r c}$, for further iterations, until a legitimate codeword is found or the affordable maximum number of iterations has been exhausted.

\section{NON-BINARY EXIT CHART ANALYSIS}

The main objective of employing EXIT charts [19], is to predict the convergence behaviour of the iterative decoder by examining the evolution of the input/output mutual information exchange between the inner and outer decoders in consecutive iterations. Denoting the mutual information between two random variables $X$ and $Y$ as $I(X ; Y)$, the average a priori information, $I_{A_{u r c}}$, at the input of the inner non-binary rate- 1 decoder and the average extrinsic information, $I_{E_{u r c}}$, at the output of the inner nonbinary rate-1 decoder can be defined as [22]

$$
\begin{aligned}
I_{A_{u r c}} & :=\frac{1}{M} \sum_{i=0}^{M-1} I\left(V_{i} ; \mathbf{A}_{\text {urc }}(i)\right), \\
I_{E_{u r c}} & :=\frac{1}{M} \sum_{i=0}^{M-1} I\left(V_{i} ; \mathbf{E}_{\text {urc }}(i)\right),
\end{aligned}
$$

where $V_{i}$ is an $L$-ary random variable representing the $i^{t h}$ integer symbol, $v_{i}$, at the input of the rate- 1 encoder of Figure 1 and $M$ is the total number of legitimate symbols $v_{i}$. Note that $\mathbf{A}_{u r c}(i)$ and $\mathbf{E}_{\text {urc }}(i)$ are vectors of random variables corresponding to the $i^{\text {th }}$ row of the matrices $\mathbf{A}_{u r c}$ and $\mathbf{E}_{u r c}$, respectively. The transfer characteristic $T_{u r c}$ of the inner rate- 1 decoder is a function of $I_{A_{u r c}}$ and $E_{b} / N_{0}$ expressed as $I_{E_{u r c}}=T_{u r c}\left(I_{A_{u r c}}, E_{b} / N_{0}\right)$. Similarly, the average a priori information, $I_{A_{l d p c}}$, at the input of the outer non-binary LDPC decoder and the average extrinsic information, $I_{E_{l d p c}}$, at the output of the outer non-binary LDPC decoder can be defined using Equation (10), where the subscript $u r c$ is replaced with the subscript $l d p c$. The transfer characteristic $T_{l d p c}$ of the outer non-binary LDPC decoder is given by $I_{E_{l d p c}}=$ $T_{l d p c}\left(I_{A_{l d p c}}\right)$, which does not depend on the $E_{b} / N_{0}$ values. The exchange of extrinsic information in the system schematic of Figure 2 is visualised by plotting the extrinsic information transfer characteristics of the inner non-binary rate-1 decoder and the outer non-binary LDPC decoder in a joint diagram. This diagram is known as the Extrinsic Information Transfer (EXIT) chart [19]. As seen in Figure 2, the outer LDPC decoder's 
extrinsic output $I_{E_{l d p c}}$ becomes the inner rate-1 decoder's a priori input $I_{A_{u r c}}$, which is usually represented on the $x$-axis of the EXIT chart. Similarly, on the $y$-axis of the EXIT chart, the inner rate-1 decoder's extrinsic output $I_{E_{u r c}}$ becomes the outer LDPC decoder's a priori input $I_{A_{l d p c}}$, as seen in Figure 2.

The mutual information $I\left(V_{i} ; \mathbf{A}_{u r c}(i)\right)$ in Equation (10) can be expressed as $[20,22]$

$$
\begin{aligned}
I\left(V_{i} ; \mathbf{A}_{u r c}(i)\right)= & \sum_{v_{i}=0}^{L-1} \int_{\mathrm{a}_{u r c}(i)} p\left(\mathrm{a}_{u r c}(i) \mid v_{i}\right) p\left(v_{i}\right) \\
& \cdot \log _{2}\left(\frac{p\left(\mathrm{a}_{u r c}(i) \mid v_{i}\right)}{p\left(\mathrm{a}_{u r c}(i)\right)}\right) d \mathrm{a}_{u r c}(i),
\end{aligned}
$$

with

$$
p\left(\mathrm{a}_{u r c}(i)\right)=\sum_{v_{i}=0}^{L-1} p\left(\operatorname{a}_{u r c}(i) \mid v_{i}\right) p\left(v_{i}\right),
$$

and the a priori probabilities $p\left(v_{i}\right)$ for the indices $v_{i}$. The $L$ dimensional integration in (11) can be evaluated numerically, where the pdf $p\left(\mathrm{a}_{u r c}(i) \mid v_{i}\right)$ may be obtained analytically by generating $\mathbf{A}_{\text {urc }}(i)$ according to the procedure proposed in [27], assuming that the $\log _{2}(L)$ bits corresponding to $V_{i}$ are not independent. The term, $I\left(V_{i} ; \mathbf{E}_{u r c}(i)\right)$ can also be expressed using Equations (11) and (12), where $\mathrm{a}_{u r c}(i)$ is replaced with $\mathrm{e}_{\text {urc }}(i)$. This requires the determination of the distribution of $p\left(\mathrm{e}_{u r c}(i) \mid v_{i}\right)$ by means of Monte Carlo simulations and computing an $L$-dimensional histogram $[19,20]$. However, a more efficient computation of non-binary EXIT functions was proposed in [21] that requires neither the computation of the $L$-dimensional histogram nor the evaluation of the $L$-dimensional integration in Equation (11). It was shown in [21] that by averaging over a sufficiently large number of length- $K_{l d p c}$ blocks, the mutual information $I_{E}$ can be estimated as

$$
I_{E}=-H\left(V_{1}\right)+E\left\{\frac{1}{K_{l d p c}} \sum_{i=0}^{K_{l d p c}-1} \sum_{l=0}^{L-1} e_{k}^{l}\right\}
$$

where $e_{k}^{l}$ refers to the element at the cross-over point of the $k^{t h}$ row and $l^{t h}$ column of the matrices $\mathbf{E}_{u r c}$ or $\mathbf{E}_{l d p c}$ and the entropy $H\left(V_{1}\right)$ can be readily determined from the a prior $i L$-ary symbol distributions $p\left(v_{i}\right)$. For example, if we have $p\left(v_{i}=l\right)=1 / L$, for $l=0,1, \ldots, L-1$, (i.e. equiprobable $L$-ary symbols), then arrive at $H\left(V_{1}\right)=-\log _{2}(L)$.

Figure 3-a shows the EXIT chart of the symbol-based LDPCcoded STBC-SP scheme of Figures 1 and 2 in combination with $L=4$ and the $\frac{1}{2}$-rate outer LDPC code [9] defined over $G F(4)$, when operating at a signal-to-noise ratio (SNR) of $0.5 \mathrm{~dB}$ and using $I_{\text {int }}=3$ internal LDPC iterations as well as the system parameters outlined in Table I. This is referred to as Scheme 1. Figure 3-a also shows the EXIT chart of an identicalthroughput $1 / 2$ bit-per-symbol (BPS) bit-based LDPC-coded STBC-SP scheme in combination with the $\frac{1}{2}$-rate outer LDPC code defined over $G F(2)$. Binary EXIT charts [19] are employed for studying the convergence behaviour of bit-based schemes. Ideally, in order for the exchange of extrinsic information between the rate- 1 decoder and the outer LDPC decoder of Figure 2 to converge at a specific SNR value, the EXIT curve of the rate1 decoder recorded at the SNR value of interest and that of the outer LDPC decoder should only intersect at the point of $\left(I_{A}, I_{E}\right)=(2.0,2.0)$. If this condition is satisfied, then a socalled open convergence tunnel [19] appears in the EXIT chart. The narrower the tunnel, the closer the system's performance to the channel capacity and hence in the spirit of Shannon's information theory more iterations are required for reaching the $(2.0,2.0)$ point.

Observe in Figure 3-a that the symbol-based Scheme 1 of Table I exhibits an open convergence tunnel at $\mathrm{SNR}=0.5 \mathrm{~dB}$, while the equivalent bit-based scheme requires higher SNR values, before an open convergence tunnel can be formed. This

\begin{tabular}{|l||c|c|c|}
\hline \multicolumn{1}{|c||}{} & Scheme 1 & Scheme 2 & Scheme 3 \\
\hline Sphere Packing constellation size & $L=4$ & $L=8$ & $L=16$ \\
\hline No. of transmitters & \multicolumn{4}{|c|}{2} \\
\hline No. of receivers & \multicolumn{3}{|c|}{ Correlated Rayleigh Fading } \\
\hline Channel & \multicolumn{3}{|c|}{0.1} \\
\hline Normalised Doppler frequency & \multicolumn{4}{|c|}{2.5} \\
\hline Average LDPC column weight & $\frac{1}{2}$ \\
\hline LDPC coding rate & $G F(4)$ & $G F(8)$ & $G F(16)$ \\
\hline Non-Binary LDPC decoding field & $G F(4)$ & $G F(8)$ & $G F(16)$ \\
\hline Rate-1 decoding field & $1 / 2$ & $3 / 4$ & 1 \\
\hline System throughput (BPS) & \multicolumn{3}{|c|}{1488 to 12000 bits } \\
\hline LDPC Coded Blocklength & \multicolumn{3}{|c|}{}
\end{tabular}

TABLE I

SYSTEM PARAMETERS

implies that according to the predictions of the EXIT chart seen in Figure 3-a, the symbol-based Scheme 1 of Table I is expected to have a lower convergence threshold than its bit-based counterpart and hence the former will exhibit a BER turbo cliff at a lower SNR value.

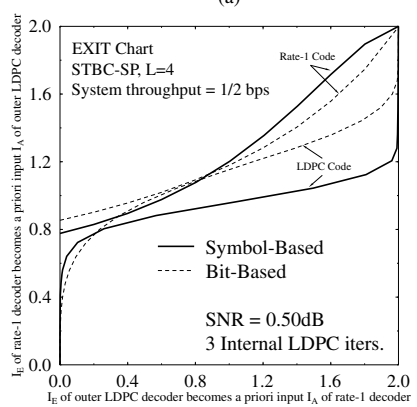

(b)

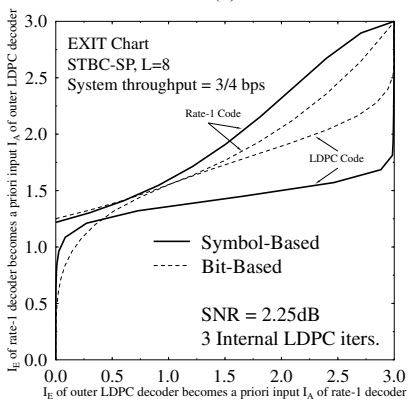

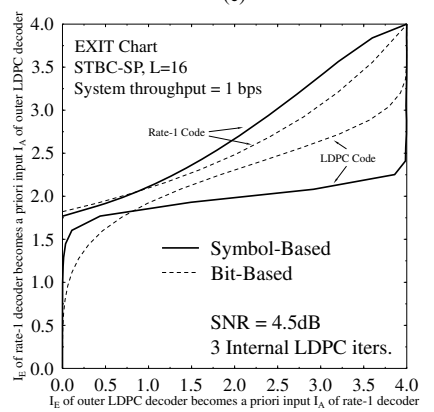

Fig. 3. EXIT chart of a symbol-based and a bit-based LDPC-coded STBC-SP schemes in combination with the $\frac{1}{2}$-rate outer LDPC codes defined over $G F(q)$ and $G F(2)$, respectively, using three internal LDPC iterations and the system parameters outlined in Table I. (a) Scheme 1. (b) Scheme 2. (c) Scheme 3.

Figures 3-b and 3-c show the EXIT charts of the symbolbased Scheme 2 and Scheme 3 of Table I, when using $I_{\text {int }}=3$ internal LDPC iterations and operating at SNRs of $2.25 \mathrm{~dB}$ and $4.50 \mathrm{~dB}$, respectively. Figures 3-b and 3-c also show the EXIT charts of the equivalent-throughput bit-based schemes. Observe in Figure 3-c that although both the symbol-based and bit-based schemes require similar SNR values in order to exhibit an open convergence tunnel, the symbol-based scheme exhibits a wider tunnel. Hence a lower number of iterations is needed to reach the convergence point of $\left(I_{A}, I_{E}\right)=(4.0,4.0)$. These EXIT tunnel based convergence predictions are usually verified by the actual EXIT trajectory of iterative decoding as well as by the BER curves, as it will be discussed in Section V. 


\section{RESULTS AND DISCUSSION}

Without loss of generality, we considered a sphere packing modulation scheme associated with $L=4,8$ and 16 using two transmit and a single receiver antenna, in order to demonstrate the performance improvements achieved by the proposed system. All simulation parameters are listed in Table I. There are more than $L$ legitimate SP symbols in the lattice $D_{4}$ and hence the required $L \mathrm{SP}$ symbols were chosen according to the minimum energy and highest minimum Euclidean distance (MED) criterion proposed in [24].
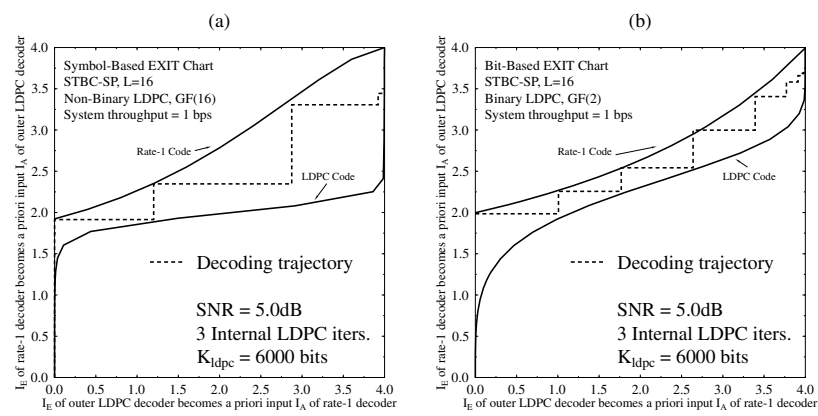

Fig. 4. Decoding trajectory of the symbol-based and bit-based $\frac{1}{2}$-rate LDPC coded STBC-SP schemes in combination with the system parameters outlined in Table I and operating at $E_{b} / N_{0}=5.0 \mathrm{~dB}$ after five joint external iterations and three internal LDPC iterations. (a) Symbol-based scheme. (b) Bit-based scheme.

Figure 4-a illustrates the actual decoding trajectory of the turbo-detected symbol-based non-binary LDPC-coded STBC-SP scheme of Figure 3-c, when operating at $\mathrm{SNR}=5.0 \mathrm{~dB}$ after $I_{\text {ext }}=5$ joint external iterations and $I_{\text {int }}=3$ internal LDPC iterations. The 'zigzag-path' seen in Figure 4-a represents the actual extrinsic information transfer between the rate-1 inner decoder and the outer non-binary LDPC decoder at SNR $=5.0 \mathrm{~dB}$ The deviation of the decoding trajectory from the prediction of the EXIT chart is due to the fact that a finite LDPC output blocklength of $K_{l d p c}=6000$ bits is employed, rendering the assumption of having Gaussian distributed symbol probabilities only approximately valid. This assumption was exploited, when creating $\mathbf{A}_{u r c}$ and $\mathbf{A}_{l d p c}$ for the sake of generating the appropriate a priori information $I_{A}$, in order to characterise the EXIT characteristics of the constituent decoders. Figure 4-b illustrates the decoding trajectory of the turbo-detected bit-based binary LDPC-coded STBC-SP scheme of Figure 3-c, when operating at $\mathrm{SNR}=5.0 d B$ after $I_{\text {ext }}=15$ joint external iterations and $I_{\text {int }}=3$ internal LDPC iterations. Observe in Figures 4-a and 4$\mathrm{b}$ that more joint external iterations are required by the bit-based scheme in order to converge than by the symbol-based scheme.

Figure 5 compares the attainable performance of both the symbol-based non-binary LDPC [9] and of the bit-based binary LDPC [6] coded STBC-SP schemes using the system parameters of Table I after $I_{\text {ext }}=5$ joint external iterations and $I_{\text {int }}=3$ internal LDPC iterations, when using an LDPC output blocklength of $K_{l d p c}=12000$ bits. The effect of employing different interleaver sizes or, equivalently, LDPC output blocklengths on the achievable performance of the symbol-based scheme is illustrated in Figure 6. More specifically, Figure 6 characterises the performance of the symbol-based $\frac{1}{2}$-rate LDPC [9] coded STBCSP schemes using the system parameters of Table I after $I_{\text {ext }}=5$ joint external iterations and $I_{i n t}=3$ internal LDPC iterations for various LDPC output blocklengths. The SNR values required for achieving a BER of $10^{-5}$ are highlighted in Figure 7 versus the system throughput for both the bit-based and symbol-based LDPC coded STBC-SP schemes using the system parameters of Table I, when using three internal LDPC iterations and an LDPC output blocklength of $K_{l d p c}=12000$ bits.

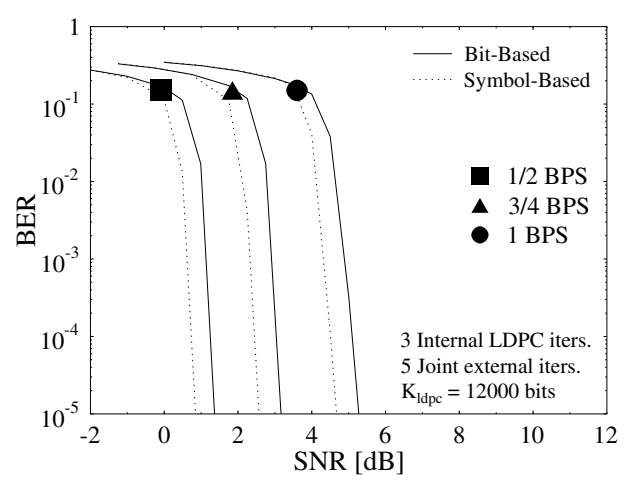

Fig. 5. Performance of symbol-based and bit-based LDPC coded STBC-SP schemes in combination with the system parameters outlined in Table I after five joint external iterations and three internal LDPC iterations, when using an LDPC output blocklength of $K_{l d p c}=12000$.

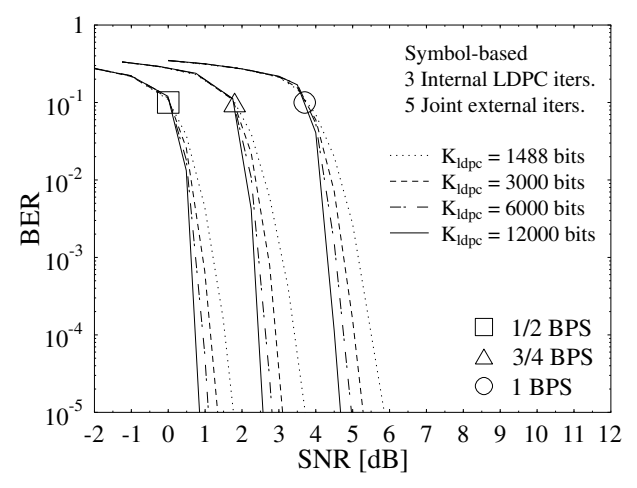

Fig. 6. Performance of symbol-based $\frac{1}{2}$-rate LDPC [9] coded STBC-SP schemes in combination with the system parameters outlined in Table I after five joint external iterations and three internal LDPC iterations while using different LDPC output blocklengths.

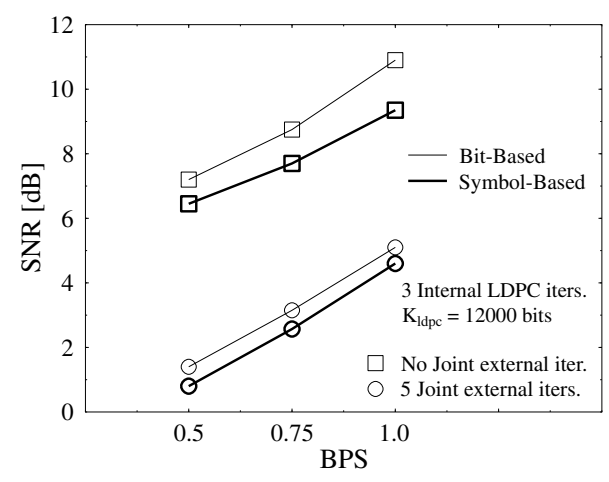

Fig. 7. SNR values required to achieve a BER of $10^{-5}$ versus different system throughput for both the bit-based and symbol-based LDPC coded STBC-SP schemes in combination with the system parameters outlined in Table I, when using three internal LDPC iterations and an LDPC output blocklength of $K_{l d p c}=12000$ bits. 


\section{CONCLUSION}

In this paper we proposed a novel symbol-based iterative scheme that exploits the advantages of non-binary LDPC codes [9], those of the rate-1 inner codes of [14] as well as those of the STBC-SP scheme of [5]. Our investigations demonstrated that attractive performance improvements may be achieved by the proposed scheme over the equivalent-throughput bit-based schemes. Subsequently, novel non-binary EXIT charts were used for studying the convergence of the proposed symbol-based scheme. By contrast, binary EXIT charts were used for exploring the convergence of the bit-based binary LDPC-coded STBCSP schemes. Again, it was demonstrated both by EXIT chart analysis and by the corresponding BER performance curves that the symbol-based scheme is capable of outperforming its bitbased counterpart and both designs had an edge over Alamouti's now classic STBC scheme dispensing with the SP-based joint design of the QPSK space-time symbols. Our future research will consider similar differentially encoded low-complexity SP designs for the sake of requiring no channel estimation as well as space-time equalised systems.

\section{REFERENCES}

[1] L. Hanzo, T. H. Liew, and B. L. Yeap, Turbo Coding, Turbo Equalisation and Space-Time Coding: for Transmission over Fading Channels. Chichester, England: John Wiley and Sons Ltd and IEEE Press, NY, USA, 2002.

[2] S. Alamouti, "A simple transmit diversity technique for wireless communications," IEEE Journal on Selected Areas in Communications, vol. 16, no. 8, pp. 1451-1458, 1998.

[3] V. Tarokh, H. Jafarkhani, and A. Calderbank, "Space-time block codes from orthogonal designs," IEEE Transactions on Information Theory, vol. 45, pp. 1456-1467, Jul 1999.

[4] B. M. Hochwald, G. Caire, B. Hassibi, and E. T. L. Marzetta, "Special issue on space-time transmission, reception, coding and signal processing," IEEE Transactions on Information Theory, vol. 49, pp. 2329-2806, Oct 2003.

[5] W. Su, Z. Safar, and K. J. R. Liu, "Space-time signal design for timecorrelated Rayleigh fading channels," in IEEE International Conference on Communications, vol. 5, (Anchorage, Alaska), pp. 31753179,2003

[6] R. Gallager, "Low density parity check codes," IEEE Transactions on Information Theory, vol. 8, pp. 21-28, Jan. 1962.

[7] C. Berrou, A. Glavieux, and P. Thitimajshima, "Near Shannon limit error-correcting coding and decoding: Turbo codes," Proceedings of the IEEE International Confrence on Communications, pp. 10641070, May 1993

[8] T. Richardson and R. Urbanke, "The renaissance of Gallager's low-density parity-check codes," IEEE Communications Magazine, vol. 41, pp. 126-131, Aug 2003.

[9] M. C. Davey and D. J. C. MacKay, "Low density parity check codes over GF(q)," IEEE Communications Letters, vol. 2, pp. 165-167, June 1998 .

[10] G. Forney, Concatenated Codes. Cambridge: MIT Press, 1966.

[11] Consultative Committee for Space Data Systems, Recommendations for Space Data System Standards: Telemetry Channel Coding, 1984

[12] S. Benedetto and G. Montorsi, "Serial concatenation of block and convolutional codes," IEE Electronics Letters, vol. 32, no. 10 pp. 887-888, 1996.

[13] S. Benedetto, D. Divsalar, G. Montorsi, and F. Pollara, "Seria concatenation of interleaved codes: performance analysis, design, and iterative decoding," IEEE Transactions on Information Theory, vol. 44, no. 3, pp. 909-926, 1998.

[14] D. Divsalar, S. Dolinar, and F. Pollara, "Serial concatenated trellis coded modulation with rate-1 inner code," in IEEE Global Telecommunications Conference, vol. 2, (San Francisco, USA), pp. 777-782, 27 Nov-1 Dec 2000.

[15] K. Narayanan, "Effect of precoding on the convergence of turbo equalization for partial response channels," IEEE Journal on Selected Areas in Communications, vol. 19, pp. 686-698, April 2001

[16] I. Lee, "The effect of a precoder on serially concatenated coding systems with an ISI channel," IEEE Transactions on Communications, vol. 49, pp. 1168-1175, Jul 2001.

[17] H. Tullberg and P. Siegel, "Serial concatenated trellis coded modulation with inner rate-1 accumulate code," in Global Telecommunications Conference, 2001. GLOBECOM '01. IEEE, vol. 2, (San Antonio, TX), pp. 936-940, 2001
[18] B. Scanavino, G. Montorsi, and S. Benedetto, "Convergence properties of iterative decoders working at bit and symbol level," in IEEE Global Telecommunications Conference, vol. 2, (San Antonio, TX), pp. 1037-1041, 2001.

[19] S. ten Brink, "Designing iterative decoding schemes with the extrinsic information transfer chart," AEÜ International Journal of Electronics and Communications, vol. 54, pp. 389-398, Nov 2000.

[20] A. Grant, "Convergence of non-binary iterative decoding," IEEE Global Telecommunications Conference, vol. 2, pp. 1058-1062, Nov 2001.

[21] J. Kliewer, S. X. Ng, and L. Hanzo, "On the computation of exit characteristics for symbol-based iterative decoding," in 4th International Symposium on Turbo Codes in connection with 6th International ITG-Conference on Source and Channel Coding, (Munich, Germany), April 2006.

[22] I. Land, P. Hoeher, and S. Gligorevic, "Computation of symbol-wise mutual information in transmission systems with $\log$ APP decoders and application to EXIT charts," in Proceedings of the International ITG Conference on Source and Channel Coding (SCC), (Erlangen, Germany), pp. 195-202, Jan 2004

[23] O. Alamri, F. Guo, M. Jiang, and L. Hanzo, "Turbo detection of symbol-based non-binary LDPC-coded space-time signals using sphere packing modulation," in IEEE Vehicular Technology Conference, vol. 1, (Dallas, USA), pp. 540-544, Sep 2005.

[24] O. Alamri, B. L. Yeap, and L. Hanzo, "A turbo detection and sphere packing modulation aided space-time coding scheme," IEEE Transactions on Vehicular Technology, vol. 56, pp. 575-582, Mar. 2007.

[25] S. Benedetto, D. Divsalar, G. Montorsi, and F. Pollara, "A softinput soft-output APP module for iterative decoding of concatenated codes," in IEEE Communications Letters, vol. 1, pp. 22-24, Jan 1997.

[26] L. Bahl, J. Cocke, F. Jelinek, and J. Raviv, "Optimal decoding of linear codes for minimizing symbol error rate," IEEE Transactions on Information Theory, vol. 20, pp. 284-287, March 1974.

[27] S. X. Ng, J. Y. Chung, and L. Hanzo, "Turbo-detected unequal protection mpeg-4 wireless video telephony using multi level coding, trellis coded modulation and space-time trellis coding," IEE Proceedings on Communications, vol. 152, pp. 1116-1124, Dec 2005. 